\section{Controlling-Lexikon}

\title{
Das Supply-Chain Operations Reference (SCOR)-Modell
}

\section{Motivation}

Das Supply Chain Management (SCM) konzentriert sich in Ausdehnung der betriebswirtschaftlichen Logistikkonzeption als strategisches Managementkonzept auf unternehmensübergreifende Wertschöpfungsnetzwerke, die sich an der Bedürfnisbefriedigung von Endkunden ausrichten. Dazu sind Entscheidungen über die Gestaltung von Geschäftsprozessen, Netzwerkstrukturen und Managementkomponenten erforderlich. Das für das SCM immanente Denken in Geschäftsprozessen erfordert eine horizontal gerichtete Sicht auf ein Unternehmen und adressiert besonders die Schnittstellen zwischen vertikal ausgerichteten Unternehmensbereichen ebenso wie zwischen den Akteuren einer Supply Chain. Die Gestaltung der Geschäftsprozesse in Supply Chains unterliegt einer Vielzahl von Einflussgrößen. Dazu zählen beispielsweise Kundengruppen, Sortimentsbereiche, Fertigungsprozesse oder auch der Grad der Arbeitsteiligkeit zwischen den Akteuren. Zudem führen Mehrfachmitgliedschaften von Unternehmen in verschiedenen Supply Chains zu einer beachtlichen Komplexität von Netzwerkstrukturen, Geschäftsprozessen und Managementkomponenten.

Um komplexe reale Probleme einer Lösung zuzuführen, bedient sich die Betriebswirtschaftslehre der Modellbildung. Referenzmodelle begünstigen eine einheitliche Terminologie, vereinfachen den konkreten Modellerstellungsprozess und den Vergleich spezifischer Modelle. Sie verfolgen damit einen Allgemeingültigkeitsanspruch und müssen den Anforderungen an Abstraktion, Robustheit gegenüber Änderungen, Flexibilität und Konsis- tenz genügen (vgl. Heusler, 2004, S. 79). Es wird zwischen Beschreibungs-, Erklärungs-, Prognose- und Entscheidungsmodellen unterschieden (vgl. Pfohl/Stölzle, 1997, S. 51 ff.).

\section{Ziele}

Das Supply Chain Operations Reference (SCOR)-Modell gilt als allgemeines Referenzmodell, welches sich zur Anwendung auf die Geschäftsprozesse unterschiedlichster Supply Chains eignet. Unmittelbare Aufgabe ist dabei die Beschreibung aller Prozesse und Aktivitäten im Zusammenhang mit der Erfüllung der Endkundennachfrage auf Basis des Supply Chain ManagementKonzepts (vgl. Huang/Sheoran/Keskar, 2005, S. 378 ff.). Das SCOR-Modell wurde auf Initiative des 1996 gegründeten Supply Chain Council (SCC) mit derzeit fast 1000 Mitgliedsorganisationen aus Wissenschaft und Praxis entworfen. Als Ergebnis kontinuierlicher Weiterentwicklungen und Erweiterungen liegt mittlerweile die Version 7.0 vor. Wurzeln des Modells finden sich im Konzept des Business Process Reengineering sowie in weiteren Konzepten und Methoden wie beispielsweise dem Benchmarking (vgl. $S C C, 2005)$.

Das SCOR-Modell bildet eine strukturierende Plattform zur Ausgestaltung spezifischer Modelle. Es empfiehlt sich als branchenübergreifender QuasiIndustrie-Standard und erleichtert somit den Austausch sowie die Zusammenarbeit von Unternehmen in Supply Chains (vgl. Huang/Sheoran/Keskar, 2005, S. 391). Nachgeordnete Ziele des Modells liegen in der Schaffung einer Grundlage für ein unternehmensübergreifendes Performance Measurement und der Suche nach Best Practi-
ce-Lösungen im SCM. Weiterhin soll das Modell die Beurteilung alternativer Supply Chain-Konfigurationen sowie die Entwicklung und Auswahl leistungsfähiger SCM-Software unterstützen (vgl. Weber, 2002, S. 197 f.).

\section{Aufbau}

Das SCOR-Modell basiert auf der Vorstellung einer integrierten Supply Chain unter Einbezug sämtlicher Lieferanten-, Produktions- und Distributionsstufen bis zum Endkunden. Es besteht aus vier hierarchisch geordneten Ebenen, von denen die oberen drei Ebenen näher spezifiziert sind. Diese differieren inhaltlich als Top-LevelProzesse, Prozesskategorien sowie Prozesselemente (vgl. Abb. 1).

Auf der obersten Ebene (Top-LevelProzesse) werden die Prozesse „Plan“, „Source“, „Make“, „Deliver“ und „Return“ unterschieden. „Plan“ adressiert alle Planungsprozesse und erhält somit einen gewissen übergeordneten Charakter. „Source“ umfasst die Beschaffungsaktivitäten. „Make“ beinhaltet die Produktionsprozesse und verwandte Aktivitäten wie die Kapazitätssteuerung. „Deliver“ bezieht sich auf die Distributionsprozesse an der Schnittstelle zu den Kunden. „Return“ spricht die Rücklaufprozesse an (vgl. Weber, 2002, S. 198 ff.).

Die fünf Top-Level-Prozesse werden auf Ebene 2 des SCOR-Modells nach verschiedenen Prozesstypen (Planning, Execution, Enable) in insgesamt 30 Prozesskategorien aufgelöst. "Make“ unterscheidet beispielsweise nach Art der Auftragsauslösung unter anderem Make-to-Stock- und Make-to-OrderProzesse. Auf dieser Ebene werden Schnittstellenprobleme und Steue- 


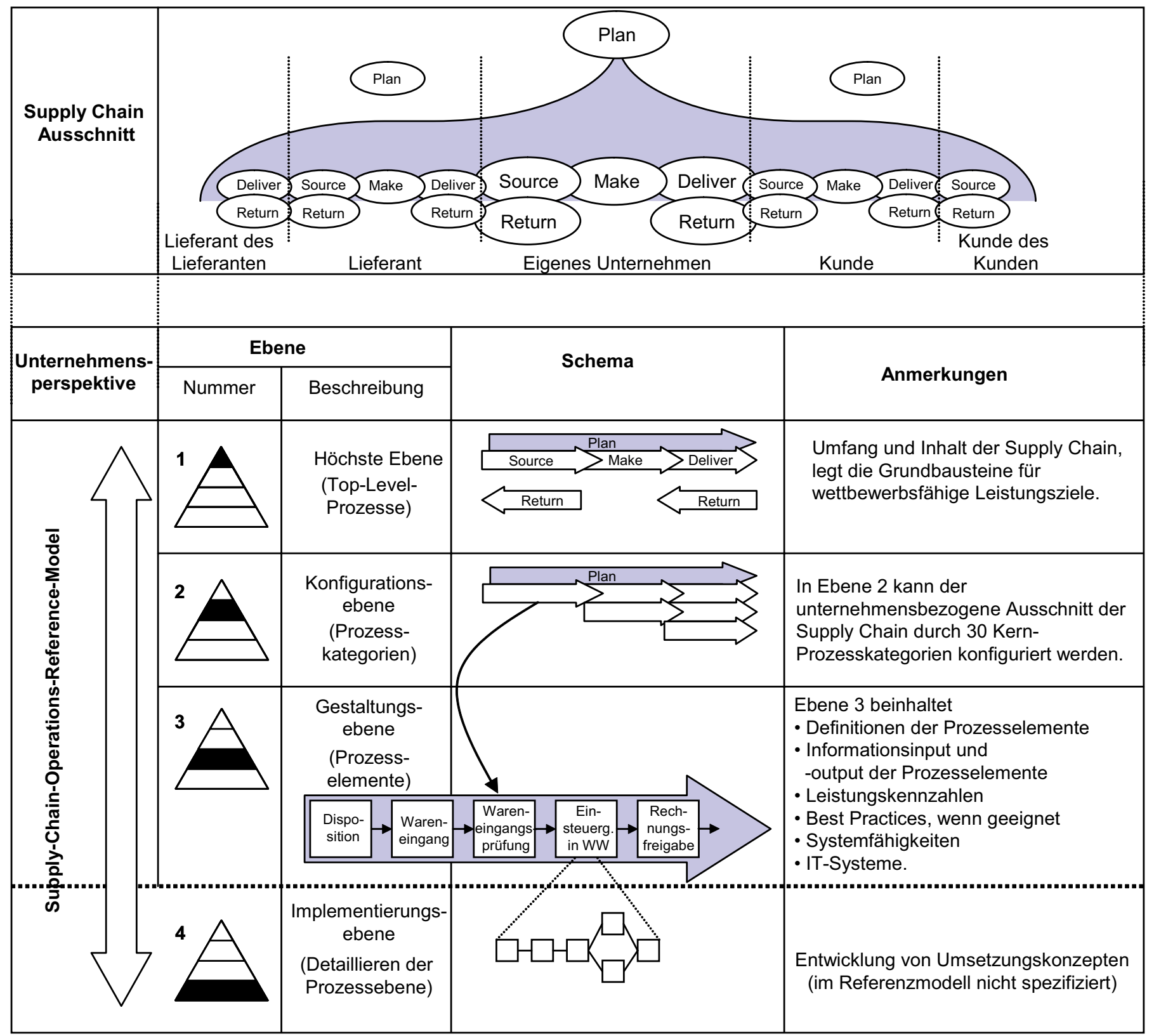

Abb. 1: Die hierarchischen Ebenen des SCOR-Modells und die Verknüpfung zum Supply Chain-Ausschnitt auf Level-1 Ebene (vgl. SCC, 2005, S. 3 und 6).

rungsaktivitäten durch den erhöhten Detaillierungsgrad und die Verknüpfung der Teilprozesse stärker sichtbar.

Die Prozesskategorien teilen sich auf Ebene 3 weiter in Prozesselemente mit definierten In- und Outputs auf. Die Prozesskategorie „Source stocked Product“ im Top-Level-Prozess „Source“ wird so in fünf Prozesselemente (Disposition der Lieferungen, Wareneingang, Wareneingangsprüfung, Einsteuerung in die betriebliche Warenwirtschaft, Freigabe der Zahlung der Lieferantenrechnung) aufgelöst. Die
Ebene 4 und gegebenenfalls weitere Ebenen sind für die anwendungsspezifische und stark implementierungsbezogene Detaillierung einzelner Prozesselemente vorgesehen und werden deshalb im Referenzmodell nicht weiter spezifiziert (vgl. SCC, 2005, S. 8 ff.).

Die unternehmensübergreifende Darstellung der Prozesse erfolgt über eine Verknüpfung der Top-Level-Prozesse der beteiligten Unternehmen einer Supply Chain. Beispielsweise sind die Top-Level-Prozesse „Deliver“ eines Lieferanten und „Source“ des Abneh- mers auf der nächsten Stufe miteinander verknüpft. Der Top-Level-Prozess „Plan“ hat einen übergeordneten Charakter, indem er jede Schnittstelle zwischen den anderen Top-Level-Prozessen anspricht und damit für eine Prozessabstimmung sorgt (vgl. $A b b .1$ ).

Mit Blick auf eine möglichst einheitliche Leistungsmessung in Supply Chains hält das SCOR-Modell Leistungskennzahlen und Best Practices bereit. Diese Systematik findet sich in der aktuellen Version 7.0 in erweiterter Form wieder (vgl. SCC, 2005, S. 8). 


\section{Kritik}

Insgesamt ermöglicht das Modell, wie auch der vielfache Praxiseinsatz zeigt, die Beschreibung von Prozessen in Supply Chains. Dies fördert die Überwindung von Schnittstellen zwischen funktional ausgerichteten Unternehmensbereichen ebenso wie zwischen verschiedenen Unternehmen in einer Supply Chain. Damit erfährt das SCM bei der Definition, der Gestaltung und Implementierung von Supply ChainProzessen eine Unterstützung (vgl. Heusler, 2004, S. 83).

Hinsichtlich der Grundanforderungen der Flexibilität und der Robustheit müssen Unternehmen individuell zwischen den Chancen und Risiken der Standardisierung, die das Modell bietet, abwägen. Über die in den oberen Ebenen weitgehend geschlossene Systematik standardisierter Prozesselemente und Schnittstellen wird das Konsistenzziel des Referenzmodells insgesamt erreicht (vgl. Weber, 2002, S. 200 f.). Mit Blick auf den Abstraktionsgrad verzichtet das Modell auf die Spezifizierung der Prozesse ab Ebene 4. Zudem ist es explizit branchenneutral ausgerichtet.

Die große Nähe zum Geschäftsprozessmanagement wirft gewisse Zweifel an der Novität der Modellgestaltung auf (vgl. Heusler, 2004, S. 83 f.). Ebenso wie viele andere Modellansätze beschränkt sich auch das SCOR-Modell auf die Deskription und verzichtet auf erklärende sowie entscheidungsunterstützende Aussagen. Zudem bezieht sich die Prozesshierarchie im Kern auf einzelne Unternehmen in der Supply Chain. Der unternehmensübergreifende Charakter wird erst durch die Verknüpfung der unternehmensbezogenen Modellspezifikationen sowie mittels übergreifender Kennzahlenstandards erreicht. Über die laufende Weiterentwicklung hinaus gibt es einzelne Veränderungsvorschläge. So könnte die Erweiterung des SCOR-Modells um Prozesse aus Marketing und Entwicklung insbesondere für Unternehmen, in denen diese Funktionen in hohem Maße unternehmensübergreifend wahrgenommen werden, zu Nutzensteigerungen führen (vgl.
Weber, 2002, S. 201). Ebenso würde die Öffnung für unternehmensspezifische Organisationsstrukturen und Kennzahlensysteme den hohen Einführungsund Anpassungsaufwand reduzieren. Schließlich wird die Bereitstellung von zusätzlichen Services durch das Supply Chain Council hinsichtlich Training und Implementierung empfohlen (vgl. $\mathrm{Hu}$ ang/Sheoran/Keskar, 2005, S. 391 ff.).

\section{Literatur}

Heusler, K. F., Implementierung von Supply Chain Management. Kompetenzorientierte Analyse aus der Perspektive eines Netzwerkakteurs, Wiesbaden 2004.

Huang, S. H./Sheoran, S. K./Keskar, H., Computer-assisted supply chain configuration based on supply chain operations reference (SCOR) model, in: Computers \& Industrial Engineering, Vol. 48 (2005) 2, S. 377394.

Pfohl, H.-Ch./Stölzle, W., Planung und Kontrolle. Konzeption, Gestaltung, Implementierung, 2. Aufl., München 1997.

Supply-Chain Council (SCC), Supply-Chain Operations Reference-model: SCOR Version 7.0 Overview. URL: www.supply-chain.org., Stand 09.05.2005.

Weber, J., Logistik- und Supply Chain Controlling, 5. Aufl., Stuttgart 2002.

Prof. Dr. Wolfgang Stölzle und Dipl.-Ök. Elias Halsband, St. Gallen
Pfohl/Stölzle

\section{Planung und Kontrolle}

Konzeption, Gestaltung, Implementierung

Von Prof. Dr. Dr. h.c. Hans-Christian Pfohl, Darmstadt, und Prof. Dr. Wolfgang Stölzle, St. Gallen

2. Auflage. 1997. X, 298 Seiten.

Gebunden $€$ 25,-

ISBN 3-8006-2161-4

(Vahlens Handbücher der Wirtschafts- und Sozialwissenschaften)

Dieses Lehr- und Handbuch ist als Lerngrundlage zur umfassenden Einarbeitung in die interdependenten Planungs- und Kontrollprobleme gedacht. Es werden alle betriebswirtschaftlich relevanten Aspekte angesprochen, über die jeder informiert sein sollte, der sich mit der Gestaltung und der Implementierung von Planungs- und Kontrollsystemen oder mit der Planung und Kontrolle der Ziele, Maßnahmen und Ressourcen für die verschiedenen Bereiche und Dimensionen des Unternehmens befasst. Der Planungs- und Kontrollprozess wird dabei sowohl als rationaler Informationsverarbeitungsprozess der Aufklärung über Handlungsvoraussetzungen, -möglichkeiten und -folgen als auch als durch Individual- und Gruppenverhalten geprägter Prozess der Konfliktaustragung und Konsensbildung verstanden.

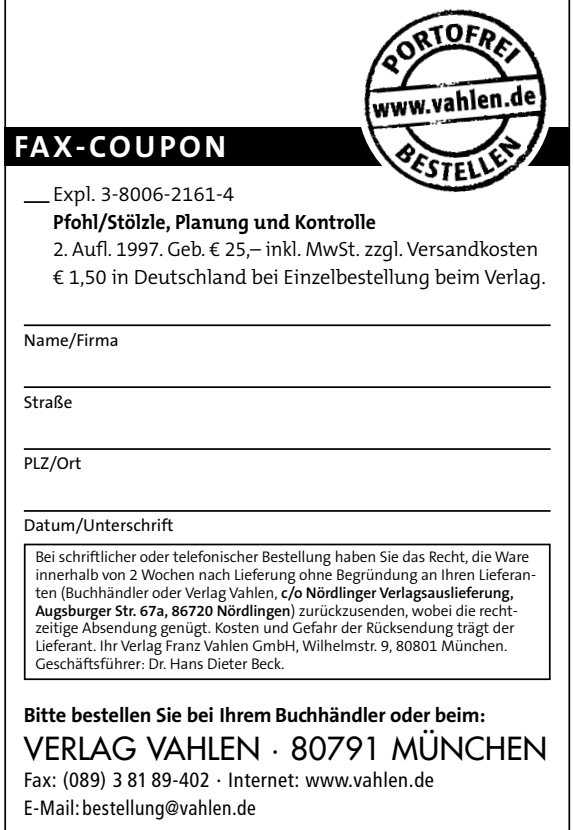

